\title{
Influence of confinement on a two-dimensional wake
}

\author{
Luca Biancofiore $^{1}$, François Gallaire ${ }^{2} \dagger$ and Richard Pasquetti ${ }^{1}$ \\ ${ }^{1}$ Laboratoire J. A. Dieudonné Université de Nice Sophia Antipolis, Parc Valrose, \\ F-06108 Nice CEDEX 02, France \\ ${ }^{2}$ EPFL/LFMI, Route Cantonale, Lausanne, Switzerland
}

(Received 10 June 2010; revised 1 June 2011; accepted 6 September 2011; first published online 21 October 2011)

\begin{abstract}
The spatio-temporal development of an incompressible two-dimensional viscous wake flow confined by two flat slipping plates is investigated by means of direct numerical simulation (DNS), using a spectral Chebyshev multi-domain method. The limit between unstable and stable configurations is determined with respect to several non-dimensional parameters: the confinement, the velocity ratio and two different Reynolds numbers, 100 and 500. The comparison of such limit curves with theoretical results obtained by Juniper (J. Fluid Mech., vol. 565, 2006, pp. 171-195) confirms the existence of a region at moderate confinement where the instability is maximal. Moreover, instabilities are also observed under sustained co-flow, in the form of a vacillating front. Using a direct computation of the two-dimensional base flow, we perform a local linear stability analysis for several velocity profiles prevailing at different spatial locations, so as to determine the local spatio-temporal nature of the flow: convectively unstable or absolutely unstable. Comparisons of the DNS and local stability analysis results are provided and discussed.
\end{abstract}

Key words: absolute/convective instability, wakes

\section{Introduction}

Coaxial injectors are often used in practical applications to mix a slow fluid flowing from the central injector with a fast fluid issuing from the external annular section. In the case of a liquid/gas interface, this process is known as air-blast atomization: when the liquid is surrounded by a high-speed coaxial gas stream with greater momentum flux, one observes a breakup and atomization of the jet which is caused by the transfer of kinetic energy from the gas to the liquid (Lasheras \& Hopfinger 2000). Besides its applications to two-phase flow chemical reactors or food processing, it is particularly efficient for fuel injectors in gas turbines, jet engines, or even liquid propellant rocket engines, where liquid oxygen is atomized by a high-speed annular coaxial hydrogen gas jet. In this process, the efficiency of the atomization and of the mixing of the liquid oxygen with the hydrogen gas crucially determines the formation of a stable flame front. It has been known for some time, without knowing the underlying mechanism, that recessing the central tube of the coaxial injector improves mixing of the two streams (Gill 1978).

$†$ Email address for correspondence: francois.gallaire@epfl.ch 
Juniper \& Candel (2003) and Juniper (2006) have analysed the influence of confinement on a channelled plane wake to mimic the effect of recessing the exit of the central injector within the outer channel. They have modelled the channelled plane wake by a slow inner stream sandwiched between two outer identical faster streams more or less confined by sidewalls. By analysing the local spatio-temporal stability properties of this inviscid parallel top-hat wake, they have discovered that the absolutely unstable nature of these flows is exacerbated when the aperture of the channel approximately equals twice the width of the wake. Broadly speaking, an absolute instability is able to withstand the mean downstream advection and can give rise to self-sustained oscillations (Huerre \& Monkewitz 1990). According to this scenario, the conclusions of Juniper (2006) lead to the expectation that the global instability of wakes is favoured by a confinement of the same size as the width of the wake, giving birth to vortex shedding even in the presence of consistent co-flow. The confinement-mediated absolute instability was interpreted as the main reason for the increased mixing in the presence of a recess.

The tendency towards absolute instability was attributed to a resonance between outer and inner branches of the dispersion relation and confirmed in several variations of parallel wake flow models, including finite shear thickness (Juniper 2008; Rees \& Juniper 2010), viscous effects (Rees \& Juniper 2010) and a round geometry (Juniper 2008). An alternative interpretation was given by Healey (2007), based on the transverse propagation and reflection on the wall of instability waves. These last papers completed the series of variants of absolute/convective analysis of planar and axisymmetric wakes and jets, including for example the influence of the shear layer thickness (Monkewitz 1988), Reynolds number (Monkewitz 1988), density ratio (Yu \& Monkewitz 1990), viscosity ratio (Sevilla, Gordillo \& Martinez-Bazan 2002), surface tension (Leib \& Goldstein 1986), compressibility (Lesshafft \& Huerre 2007; Meliga, Sipp \& Chomaz 2010) and added swirling flow component (Loiseleux, Chomaz \& Huerre 1998).

As recognized early by Chomaz, Huerre \& Redekopp (1991), the existence of a finite region of absolute instability is a necessary condition for global instability. The concept of absolute/convective transition was originally associated with cylinder wakes. Following Koch (1985) and Triantafyllou, Triantafyllou \& Chryssostomodis (1986), Monkewitz (1988) and Monkewitz \& Nguyen (1987) demonstrated that the well-known von Kármán vortex street could be associated with a pocket of absolute instability located in the lee of the cylinder. This was confirmed by Hannemann \& Oertel (1989) and Hammond \& Redekopp (1997) in blunt body wakes as well as in the related situation of obstacle-free wakes pioneered by Triantafyllou \& Karniadakis (1990) and more recently studied by Pier \& Huerre (2001) and Chomaz (2004). Instead of computing the dynamics of the whole flow around the cylinder, the cylinder region was cut away and the flow computed in a semi-infinite domain, prescribing at the inlet either the axial velocity profile measured just behind the cylinder or a prescribed wake profile.

It was shown subsequently that, in many cases, the self-sustained oscillations reached saturation in the form of a so-called nonlinear global mode, described analytically by Pier, Huerre \& Chomaz (2001) and Couairon \& Chomaz (1999) in the context of weakly non-parallel flows (i.e. flows whose variations in the longitudinal direction are slow compared to the dominant wavelengths of the instability). Besides its qualitative predictive power, the weakly non-parallel nonlinear front theory can also predict quantitatively the vortex shedding frequency, depending on the nature of the instability of the inlet profile. If the flow is absolutely unstable at the inlet, the 
shedding frequency is given by its absolute frequency (see Couairon \& Chomaz 1999); otherwise, it is given by the absolute frequency prevailing at the location of the onset of the absolute region (see Pier et al. 2001). It is remarkable that these coherent oscillating structures, though fully nonlinear, are governed by a linear frequency selection criterion. The weakly non-parallel theory has given satisfying results for different spatially evolving open flows, as synthetic wakes (Pier \& Huerre 2001), spiral vortex breakdown (Gallaire et al. 2006), detached boundary layers (Marquillie \& Ehrenstein 2003) or axisymmetric wakes behind afterbodies (Meliga et al. 2010).

The purpose of the present paper is to check the validity of this scenario in confined planar wakes by relaxing the parallel flow assumption using direct numerical simulation (DNS). Such an approach, to the best of our knowledge, has only recently been considered by Tammisola et al. (2011) in the range of Reynolds number $100<R e<400$. By comparing two values of confinement $(h=1$ and $h=9$; see $\S 2.1$ for the definition of $h$ ), no destabilizing effect due to confinement was discovered in their global linear and nonlinear simulations. Note also that Tammisola et al. (2011) impose no-slip boundary conditions at the walls, in contrast to our analysis where free-slip conditions are considered in order to make the link with available theoretical predictions. In the unconfined setting, our approach is similar to those of Hammond \& Redekopp (1997) and Chomaz (2004), or that of Pier \& Huerre (2001), who added an extra pressure gradient.

The configuration considered in this paper, where two co-flowing fluid streams meet and mix with each other, differs in several respects from the wake behind an obstacle. In the case of the flow behind an obstacle the velocity difference cannot be adjusted, and crucially depends on the confinement ratio through the imposed acceleration around the obstacle. The shear layer thickness depends on the geometry of the obstacle and the exact location of the detachment points. For all these reasons, the results on wakes behind solid obstacles cannot be directly applied to confined co-flow wakes. Still, a brief overview of confined wakes past an obstacle is useful, as they benefit from a more comprehensive literature. In their experiments of the weakly confined $(h>4)$ wake of a circular cylinder at $40<R e<140$ (Re being based on the cylinder diameter), Shair et al. (1963) found that the critical Reynolds number, $R e_{c}$, associated with the transition between the steady and the unsteady regimes, increases with the confinement. This stabilizing influence was confirmed by the experiments conducted by Coutanceau \& Bouard (1977) on confined cylinder wakes for $h>5.67$. In a recent paper, Sahin \& Owens (2004) increased the confinement up to $h=0.11$ and discovered that for higher blockage ratios the influence of confinement is more subtle. While $R e_{c}$ increases with confinement up to $h>1$, confinement is destabilizing in the range $0.43<h<1$, before recovering a stabilizing influence for $0.19<h<0.43$ and revealing, at these strong confinements, more complex dynamics than supercritical Hopf bifurcation. The stabilizing effect shown by Sahin \& Owens (2004) for $h>1$ was confirmed by the recent experimental study of Rehimi et al. (2008), conducted on confined wakes $(h=2)$ past a circular cylinder, who found $R e_{c}=108$, compared to the classical $R e_{c}=47$ for unconfined cylinder wakes (see Williamson 1996 for a comprehensive review).

Using DNS, we study the influence of confinement on the stability of plane wakes. The objective is to determine whether the theoretical analyses of Juniper (2006, 2007, 2008), which show a strong effect of confinement on spatio-temporal stability properties of the flow, can be confirmed by our more realistic DNSs. Using DNS does indeed allow relaxation of two restrictive hypotheses of the theoretical studies: the absence of viscosity and the parallel character of the flow. The outline of 


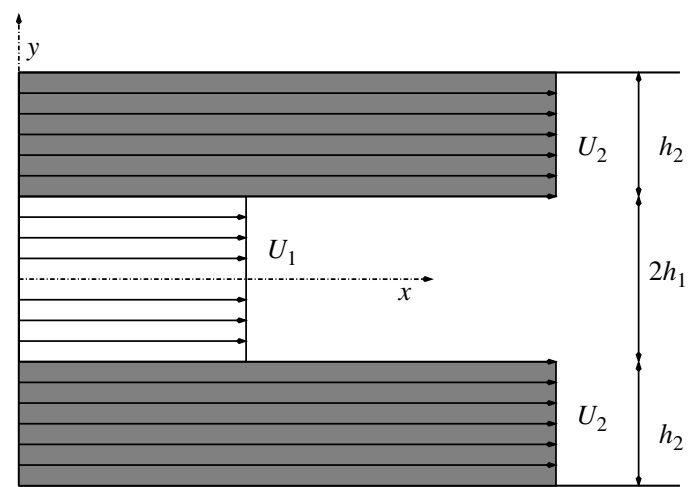

FIGURE 1. Sketch of the inlet velocity profile.

the paper is as follows: $\S 2$ describes the flow configuration and gives some details on the numerical method implemented in the DNS. The global stability region in the velocity ratio/confinement ratio plane is examined in $\$ 3$ and compared to the convective/absolute transition prevailing at the inlet. An analysis of the results is carried out in $\S 4$, where we focus on the non-parallel variations of the base flow. Finally, conclusions are provided in $\S 5$.

\section{Flow configuration and numerical method}

\subsection{Physical problem}

Let us consider the flow of a viscous fluid in a two-dimensional channel, using as in Juniper (2006) a top-hat velocity profile as inlet condition and free-slip boundary conditions at the side walls: see figure 1 .

At the inlet of the channel a flow with thickness $2 h_{1}$ and uniform velocity $U_{1}$ is sandwiched between two identical flows with thickness $h_{2}$ and uniform velocity $U_{2}$. As reference velocity and length scales, we choose the average velocity $\left(U_{1}+U_{2}\right) / 2$ and the half-thickness of wake $h_{1}$, respectively. The dimensionless parameters are then the Reynolds number $R e=\left(U_{1}+U_{2}\right) h_{1} / 2 v$, the confinement parameter $h=h_{2} / h_{1}$ and the velocity ratio $\Lambda=\left(U_{1}-U_{2}\right) /\left(U_{1}+U_{2}\right)$. For a wake, the velocity ratio is negative, $\Lambda<0$, and $-1<\Lambda<0$ corresponds to co-flow, while $\Lambda<-1$ characterizes counterflow $\left(U_{1}<0\right)$. The dimensionless entry profile, at $x=0$, is given by

$$
\begin{gathered}
U(y)=1+\Lambda \text { for } 0 \leqslant|y|<1, \\
U(y)=1-\Lambda \text { for } 1<|y| \leqslant 1+h .
\end{gathered}
$$

The study has been carried out for various values of $(\Lambda, h)=[-1.17,-0.40] \times[0.2,9]$, and two different Reynolds numbers: $R e=100$ and $R e=500$.

\subsection{Numerical model}

In dimensionless form, the Navier-Stokes system can be written:

$$
\begin{gathered}
D_{t} \boldsymbol{u}=-\nabla p+R e^{-1} \Delta \boldsymbol{u} \quad \text { in } \Omega \times\left(0, t_{f}\right), \\
\boldsymbol{\nabla} \cdot \boldsymbol{u}=0, \\
B(\boldsymbol{u})=\boldsymbol{g} \quad \text { on } \Gamma \times\left(0, t_{f}\right), \\
\left.\boldsymbol{u}\right|_{t=0}=\boldsymbol{u}_{0} \quad \text { in } \Omega,
\end{gathered}
$$




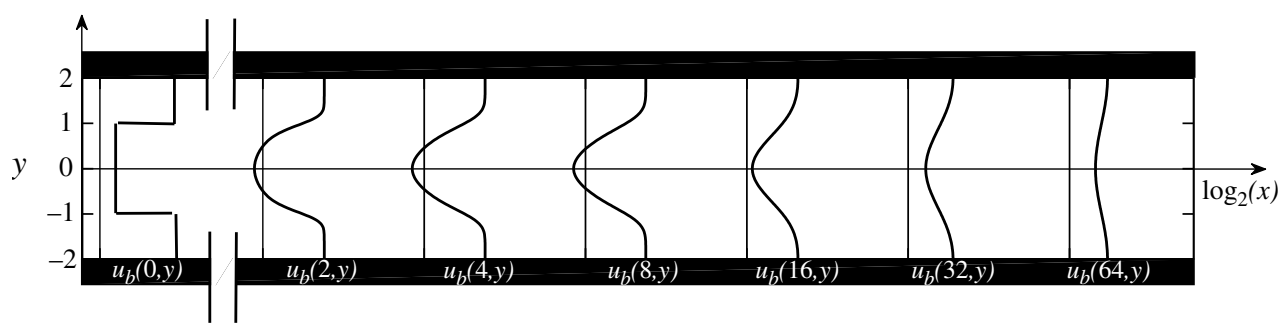

FIGURE 2. Profiles of the streamwise component of the base flow velocity $\boldsymbol{u}_{b}$, assuming freeslip conditions at the walls of the channel. The profiles have been computed for a confinement ratio $h=1$, a velocity ratio $\Lambda=-0.905$ and the Reynolds number $R e=100$. The $x$ direction is represented in $\log _{2}$-scale and the thin vertical lines represent the zero velocity. Note that although the initial configuration at $x=0$ is a co-flow, a counterflow is present in a consistent part of the domain.

where $t$ (respectively $t_{f}$ ) is the (respectively final) time, $D_{t}$ the material derivative, $\Omega$ the computational domain and $\Gamma$ its boundary, $\boldsymbol{u}=(u, v)$ the velocity and $p$ the pressure. The boundary operator $B$ and the function $g$ are used to express the boundary conditions. The domain is rectangular, that is, $\Omega=(0, L) \times(-H, H)$, where $H=1+h$, and its length must be such that $L \gg 1$ : see figure 2 .

A domain decomposition technique with conforming meshes is used in the elongated longitudinal direction, and in each subdomain we use a Chebyshev collocation approximation in both $x$-streamwise and $y$-crossflow directions. Between the different non-overlapping subdomains, say $\Omega_{s}, 1 \leqslant s \leqslant S$, the transmission conditions express the $C^{1}$ continuity of the numerical approximation $u_{N}$ of some unknown variable $u$, so that $u_{N}$ belongs to the functional space $V_{N}$ such that

$$
V_{N}=\left\{v \in C^{1}(\Omega),\left.v\right|_{\Omega_{S}} \circ f_{s} \in \mathbb{P}_{N}(-1,1)^{2}, s=1 \cdots S\right\}
$$

where $f_{s}:(-1,1)^{2} \rightarrow \Omega_{s}$ maps the reference domain to the physical one and with $\mathbb{P}_{N}$ for the set of polynomials of degree $N_{x}$ in $x$ and $N_{y}$ in $y$. More precisely, in each subdomain $u_{N}$ is formulated as

$$
\left.u_{N}\right|_{\Omega_{s}} \circ f_{s}=\sum_{i=0}^{N_{x}} \sum_{j=0}^{N_{y}} u_{i j}^{s} l_{i} l_{j}
$$

where $l_{i}$ and $l_{j}$ are the Lagrange polynomials based on the Chebyshev-Gauss-Lobatto points $-\cos \left(\pi i / N_{x}\right), 0 \leqslant i \leqslant N_{x}$, and $\left.-\cos \left(\pi j / N_{y}\right)\right), 0 \leqslant j \leqslant N_{y}$, respectively. The collocation method forces $u_{N}$ to solve the partial differential equation at all internal collocation points, i.e. such that $0<i<N_{x}$ and $0<j<N_{y}$, whereas the boundary conditions are imposed at the boundary grid-points: see e.g. Peyret (2001) for details.

We use a backward second-order finite difference approximation of the material (Lagrangian) time derivative, and the temporal scheme is then based on the following implicit time discretization of the momentum equation (the subscript $N$ is dropped for simplicity in the notation):

$$
\frac{3 \boldsymbol{u}^{n+1}-4 \tilde{\boldsymbol{u}}^{n}+\tilde{\boldsymbol{u}}^{n-1}}{2 \tau}=-\nabla p^{n+1}+\operatorname{Re}^{-1} \Delta \boldsymbol{u}^{n+1}
$$

with $n$ for the time index, $\tau$ for the time step and where $\boldsymbol{u}^{n+1} \approx \boldsymbol{u}\left(\boldsymbol{x}, t_{n+1}\right)$, whereas $\tilde{\boldsymbol{u}}^{n} \approx \boldsymbol{u}\left(\boldsymbol{\chi}\left(t_{n} ; \boldsymbol{x}, t_{n+1}\right), t_{n}\right)$ with $\boldsymbol{\chi}\left(t ; \boldsymbol{x}, t_{n+1}\right)$ for the characteristic curve that originates from $x$ at time $t_{n+1}$. 
To compute $\boldsymbol{u}^{n+1}$ and $p^{n+1}$ three steps are involved (Cousin \& Pasquetti 2004).

(a) A transport step, based on an operator integration factor (OIF) semi-Lagrangian method. This step amounts to solving pure transport problems to compute the $\tilde{\boldsymbol{u}}^{k}$, $k=n-1, n$ : see e.g. Maday, Patera \& Ronquis (1990) and Xu \& Pasquetti (2001) for details. It is handled explicitly using the fourth-order Runge-Kutta algorithm.

(b) An implicit diffusion step. Given the $\tilde{\boldsymbol{u}}^{k}$ and with the pressure expressed at $t_{n}$ (Goda scheme), a provisional velocity, say $\boldsymbol{u}^{\star}$, may be computed by solving two scalar elliptic problems. To this end we use a Schur complement technique, i.e. we first compute the subdomain interface values and then in each subdomain the resulting matrix equations are solved by using a fast diagonalization method (see Borchers et al. 1998). Note, however, that to obtain matrix equations we assume that each mapping $\boldsymbol{f}_{s}$ may be expressed as the product of two one-dimensional mappings. Moreover, using conforming meshes implies that the $y$-mappings are the same in all subdomains.

(c) A projection step, to update the pressure and obtain a velocity field $\boldsymbol{u}^{n+1}=$ $\boldsymbol{u}^{\star}+\nabla \varphi, \varphi$ such that $\boldsymbol{\nabla} \cdot \boldsymbol{u}^{n+1}=0$ at the inner grid-points. In order to obtain a well-posed discrete problem, i.e. with unique solution up to a constant and avoid 'pressure spurious modes', the pressure correction, $\varphi=p^{n+1}-p^{n}$, is computed with the so-called unique grid $\mathbb{P}_{N}-\mathbb{P}_{N-2}$ approximation (Azaiez, Fikri \& Labrosse 1994). In the monodomain case, this means that the polynomial degree for the pressure is two degrees less than for the velocity components and that $\varphi$ is only defined by its inner grid-point values, so that no pressure boundary conditions are required. As detailed in Cousin \& Pasquetti (2004), such an approach can be easily extended to the present one-dimensional domain decomposition. The resolution for $\varphi$ is again based on a Schur complement technique and, in each subdomain, on the fast diagonalization method.

The solver is parallelized (one subdomain per processor) and for each subdomain the computations are vectorized. Recently, it was successfully used for the large-eddy simulation of two challenging physical problems (see Minguez, Pasquetti \& Serre 2008; Pasquetti 2010).

\subsection{Boundary conditions}

At $y= \pm H$ we use a standard free-slip boundary condition. As detailed below, the conditions used at the inlet and the outlet of the computational domain are more subtle. In particular, the choice of a relevant outflow boundary condition (OBC) is required in the present study.

The inflow profile $U$ described in the introduction displays a discontinuity at $y= \pm 1$, which needs to be smoothed out since we use a spectral method. In order to avoid or at least weaken the Gibbs phenomenon, we use a filtered entry profile, regularizing the initial profile according to:

$$
\widetilde{U}_{i}=\frac{1}{4}\left(U_{i-1}+2 U_{i}+U_{i+1}\right),
$$

where $U_{i}$ (and $\widetilde{U}_{i}$ ) are the values at the grid-points of the $y$-axis. In the frame of a Fourier spectral approximation, it can be shown that this smoothing in physical space is equivalent to a raised cosine filtering; see e.g. Pasquetti, Bwemba \& Cousin (2008). Note that once applied to the profile in (2.1), the filter changes only the values at the grid-points next to $y= \pm 1$.

At the downstream end of the computational domain $(x=L)$ a non-reflection OBC is implemented. Both the so-called $u-\omega$ and $u-v$ exit conditions (Forestier, Pasquetti 
\& Peyret 2000) have been used, and we have adopted the first one for accuracy reasons. The $u-\omega$ condition implies that the component normal to the outlet, $u$, and the vorticity, $\omega=\partial_{x} v-\partial_{y} u$, are transported at the average fluid velocity $\bar{U}$ :

$$
\left(\partial_{t}+\bar{U} \partial_{x}\right) u=\left(\partial_{t}+\bar{U} \partial_{x}\right) \omega=0 .
$$

As a consequence of the global coupling introduced by the spectral method, it is important to 'localize' the effect of the OBC. To this end, using the method of characteristics, these conditions are implemented as follows:

$$
\begin{aligned}
u^{n+1}\left(L, t_{n+1}\right) & =u^{n}\left(L-\tau \bar{U}, t_{n}\right), \\
\omega^{n+1}\left(L, t_{n+1}\right) & =\omega^{n}\left(L-\tau \bar{U}, t_{n}\right) .
\end{aligned}
$$

Note that the $u$-profile is, moreover, slightly adjusted to preserve the flow-rate and that, as a result of (2.12), (2.13) amounts to implementing a Neumann condition on $v$ (again using a Lagrangian approach).

In the most critical cases, the $u-\omega$ OBC has been compared with a special treatment in an additional subdomain, say the sponge subdomain, where the flow is smoothly enforced to the constant mean flow velocity profile. A penalization technique is used to this end, by complementing the momentum equation with an appropriate forcing term $\boldsymbol{F}$. In the sponge subdomain, of length $\Delta L$, the divergence-free velocity solves

$$
\begin{gathered}
D_{t} \boldsymbol{u}=-\nabla p+R e^{-1} \Delta \boldsymbol{u}+\boldsymbol{F}, \\
\boldsymbol{F}=f\left(\frac{x-L}{\Delta L}\right)\left(\bar{U} \boldsymbol{e}_{x}-\boldsymbol{u}\right),
\end{gathered}
$$

where $\boldsymbol{e}_{x}$ is the unit vector in the $x$ direction. The function $f$ should be chosen to increase smoothly from 0 to its maximum value. Because an $O(1 / \tau)$ maximal value was shown by Pasquetti et al. (2008) to be well adapted to modelling an obstacle with a smoothed penalization technique, we have used in practice

$$
f(X)=\frac{0.1}{\tau} \sin ^{2}\left(\frac{\pi}{2} X\right), \quad 0<X<1 .
$$

At the end of the extended computational domain, the fluctuations with respect to the mean flow are then less than $10^{-5}$. Such an outflow treatment radically differs from the $u-\omega$ OBC. It has been implemented for such configurations for which the dependence of the flow on the exit conditions should be checked carefully.

\subsection{Initial condition}

The initial condition $\boldsymbol{u}_{0}(x, y)$ consists of the superposition of the velocity entry profile $\widetilde{U}(y)$ and a random perturbation $\boldsymbol{u}^{\prime}(x, y)$ of amplitude $10^{-3}$ :

$$
\boldsymbol{u}_{0}(x, y)=\widetilde{U}(y) \boldsymbol{e}_{x}+\boldsymbol{u}^{\prime}(x, y) .
$$

This perturbation breaks the varicose symmetry $y \leftrightarrow-y$ of the base flow velocity profile $\boldsymbol{u}_{b}(x, y)$ (figure 2), which is a steady solution of the Navier-Stokes equations (2.3)-(2.6). The base flow velocity profile prevails when the flow is stable. This breaking of the varicose symmetry allows the sinuous mode to develop faster and, thus, a gain in terms of computational time.

\subsection{Base flow}

In order to study the influence of the non-parallel feature of the base flow $\boldsymbol{u}_{b}$, we have to determine its variations. For unstable flows, displaying self-sustained oscillations, 
the base flow cannot be obtained as the end-result of the DNS. However, it may be determined by exploiting the varicose symmetry of the base flow. This amounts to taking advantage of the analogy between the varicose evolution of a wake and an asymmetric mixing layer (i.e. when the mixing layer does not lie exactly halfway between the plates), with the same flow parameters: $R e$ and $\Lambda$. Note that confined asymmetric mixing layers are globally stable in the range of parameters analysed in the paper (Healey 2009). The base flow is then simply obtained by time marching of the DNS of a mixing layer located at $y=1$ and quenched between two plates at $y=0$ and $y=H$. The free-slip condition at the lower plate eliminates any sinuous perturbation and the DNS converges to the base flow. An example of base flow is shown in figure 2 .

\subsection{Three-dimensional simulations}

With the aim of analysing the effect of the third dimension on the results, in the range of parameters analysed in this paper, several three-dimensional simulations have been carried out. The computational domain is then $(0, L) \times(-H, H) \times(-E / 2, E / 2)$, where $E$ is the thickness of the domain, and the flow is assumed to be homogeneous in the $z$ direction.

The numerical approximation for the $x$ and $y$ directions is analogous to the twodimensional case, as described in $\S 2.2$. Conversely, for the $z$ direction, a Fourier spectral approximation is implemented. As a result, (2.8) is now

$$
\left.u_{N}\right|_{\Omega_{s}} \circ \boldsymbol{f}_{s}=\sum_{i=0}^{N_{x}} \sum_{j=0}^{N_{y}} \sum_{k=-N_{z} / 2}^{N_{z} / 2} u_{i j, k}^{s} l_{i} l_{j} e_{k}
$$

where $N_{z}$ is the number of grid-points in the $z$ direction and $e_{k}$ is the trigonometric polynomial of degree $k$, i.e. $e_{k}(Z)=\mathrm{e}^{\mathrm{i} k Z}$ with $\mathrm{i}^{2}=-1$ and $Z \in(0,2 \pi)$. Of course, because $u_{N}$ is real then $u_{i j, k}^{s}$ and $u_{i j,-k}^{s}$ are complex conjugates. Here again we assume that each mapping $f_{s}$ may be expressed as the product of one-dimensional mappings and, moreover, that it is linear in the spanwise direction, so that fast Fourier transform may be used.

A standard collocation Chebyshev-Fourier-Galerkin pseudo-spectral method is implemented. Because in Fourier space $\partial_{Z} \equiv \mathrm{i} k$ and $\partial_{Z Z} \equiv-k^{2}$, and owing to the pseudo-spectral algorithm, which computes the nonlinear terms in physical space, the three-dimensional problem splits into a set of two-dimensional-like problems for the real and imaginary parts of the Fourier spectra, $0 \leqslant k \leqslant N_{z} / 2$, of the velocity and of the pressure. Each of these problems may then be solved with an approach similar to the two-dimensional one.

At the outlet of the domain, the three-dimensional form of the $u-\omega$ OBC is (Forestier et al. 2000):

$$
\left(\partial_{t}+\bar{U} \partial_{x}\right) u=0, \quad\left(\partial_{t}+\bar{U} \partial_{x}\right) \boldsymbol{\omega}_{y z}=0,
$$

where $\boldsymbol{\omega}_{y z}$ is the projection of the vorticity vector onto the outlet boundary. With $\boldsymbol{u}=(u, v, w)$ and taking into account the condition on $u$, the condition on $\boldsymbol{\omega}$ reverts to a transport condition of $\partial_{x} v$ and $\partial_{x} w$ at the mean flow velocity.

The initial condition $\boldsymbol{u}_{0}(x, y, z)$ of three-dimensional DNS is given by the final saturated state of the corresponding two-dimensional simulation $\boldsymbol{u}_{f}(x, y)$, with an additional random perturbation $\boldsymbol{u}^{\prime}(x, y, z)$ of small amplitude, typically $10^{-3}$, to induce a three-dimensional flow. 
(a)

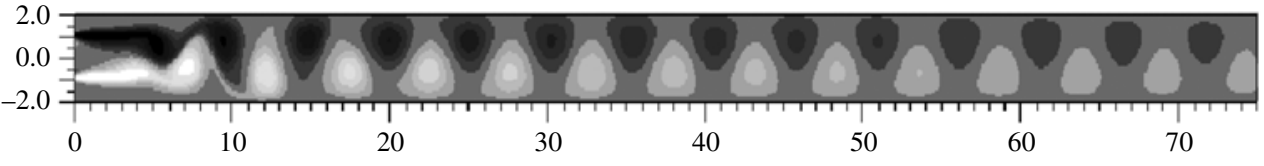

(b)
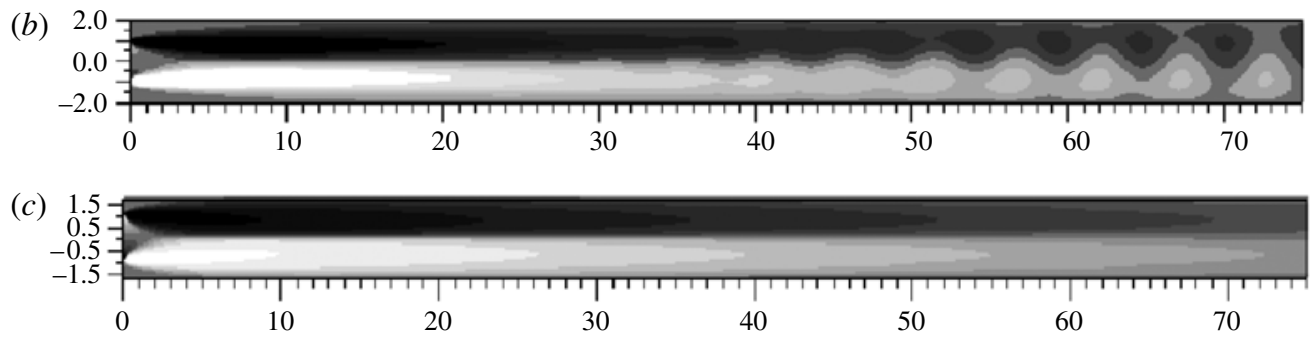

FIGURE 3. Instantaneous vorticity fields for $t=125$ and $R e=100$, for three parameter sets: (a) $h=1$ and $\Lambda=-0.739$; (b) $h=1$ and $\Lambda=-0.667$; (c) $h=0.7$ and $\Lambda=-0.739$. The negative vorticity values are shown in dark grey and the positive ones in light grey. The greyscale changes for each flow configuration.

\section{Nonlinear global stability analysis}

In $\S \S 3.1$ and 3.2 the results of the simulations are provided for $R e=100$ and $R e=500$, respectively. In $\S 3.3$, the effect of the third dimension on the stability is discussed. Generally, the length of the computational domain is $L=75$, but in order to study the effect of the exit condition on the flow stability, in some cases we used $L=34, L=101.5$ and $L=152$ to verify that the behaviours were identical. Only for the cases presenting a vacillation front have we noticed differences, as explained in $\S 3.2 .1$. For the nominal length we have generally used 8 subdomains, but for the greatest one or when the vortices are subject to a vortex pairing instability, we have also used 16 subdomains. A typical discretization in each subdomain is $N_{x}=N_{y}=100$. The time step $\tau$ is adapted to the resolution, typically $\tau=2.5 \times 10^{-3}$, and corresponds to a CFL (Courant-Friedrichs-Lewy) number $\left(:=\max (u) \tau / \min \left(h_{x}\right), h_{x}\right.$ space step in the $x$ direction) of approximately 0.7 .

$$
\text { 3.1. } R e=100
$$

Figure 3 shows the vorticity fields at time $t=125$ for three different sets of parameters $h$ and $\Lambda$. For $h=1$ and $\Lambda=-0.739$ (see figure $3 a$ ), this duration was found sufficient for the flow to settle into the saturated regime: a vortex street typical of wake flow forms at $x \approx 5$ and extends downstream. Comparing the figures allows us to identify tendencies: fixing the confinement parameter $h$, the influence of velocity ratio $\Lambda$ appears when comparing the flows obtained for $\Lambda=-0.739$ (figure $3 a$ ) and $\Lambda=-0.667$ (figure $3 b$ ). A growth in the wake inner velocity $U_{1}$ (and thereby an increase of $\Lambda$ ) pushes the perturbation downstream. Now, if we fix the velocity ratio $\Lambda=-0.739$, an increase of the confinement from $h=1$ (figure $3 a$ ) to $h=0.7$ (figure $3 c$ ) turns out to advect the perturbation. However, figure 3 represents only three instantaneous snapshots, and these tendencies have to be more systematically confirmed by a more exhaustive analysis of the velocity fields, both in space and in time, constructing spatio-temporal diagrams.

In order to better understand the spatio-temporal dynamics, the absolute value of vertical velocity component along the $x$-axis, $|v(x, 0, t)|$, may be represented on an $x-t$ diagram, as shown in figures $4(a)$ and $4(b)$, which make use of the parameters 

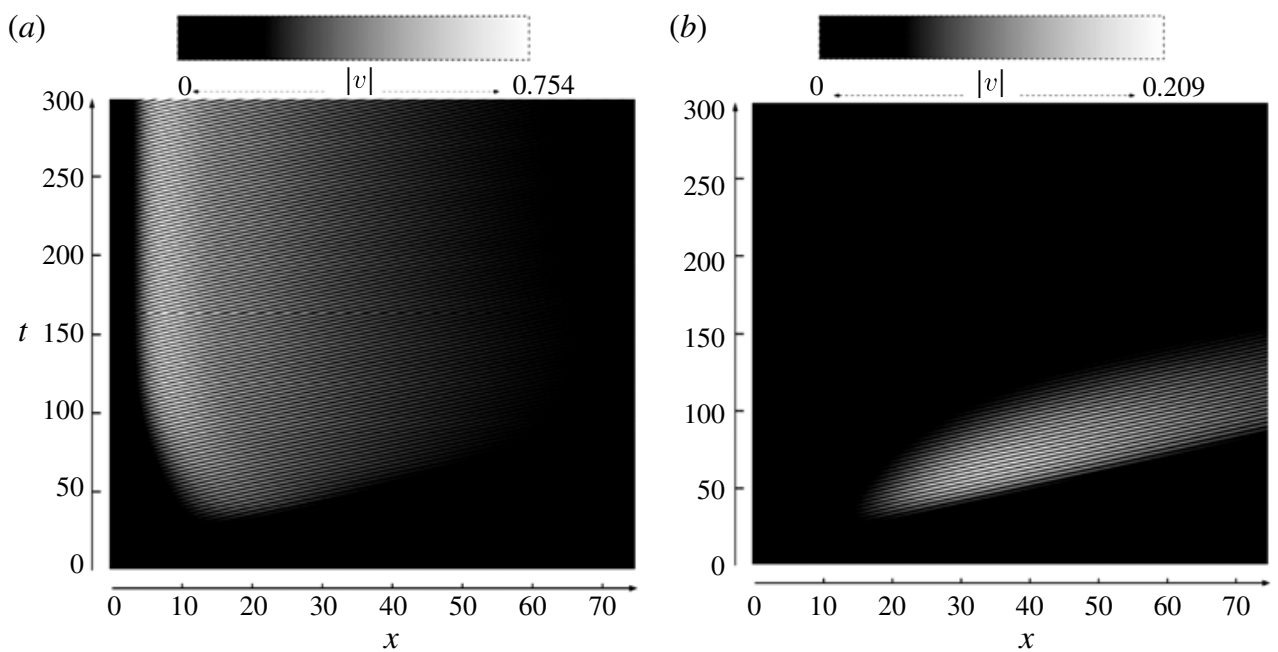

FIGURE 4. Spatio-temporal diagram of the absolute value of the velocity vertical component $v$ at $y=0$ for $h=1, \operatorname{Re}=100$, and $(a) \Lambda=-0.739,(b) \Lambda=-0.667$. (a) The flow is globally unstable: the instability persists in the domain. $(b)$ The flow is globally stable: the instability is advected downstream and disappears.

of figures $3(a)$ and $3(b)$. Figure $4(a)$ shows that for $h=1$ and $\Lambda=-0.739$, the flow is unstable and reaches an oscillating self-sustained state, or global cycle, while figure $4(b)$ shows that for the same confinement parameter $h=1$, but a faster inner fluid such that $\Lambda=-0.667$, the wake is globally stable.

For the unstable configuration (figure $4 a$ ), one observes a time-independent wave front located at $x_{f} \approx 5$. A cut in the $x-t$ plane for $x>x_{f}$, i.e. in the unstable region (in clear), shows a periodic behaviour. This oscillating behaviour with a wave front localized in space is typical of nonlinear global modes described by Pier \& Huerre (2001) for a synthetic wake or by Chomaz (2004) for a viscous wake, but for weaker Reynolds number.

In contrast to this globally unstable case, for the stable flow (figure $4 b$ ) we note the presence of a short transient, resulting from the progressive advection of an unstable wave packet. Ultimately the flow ends up becoming stable, i.e. the perturbation decreases to zero everywhere. The limit between the black part (where the flow comes back to the rest state) and the 'striped' part represents the wave front (see Chomaz 2005). If this wave front progresses with a positive velocity, as shown in figure 4(b), and moves downwards to the outlet of the domain, the flow tends to be stable in the whole domain. This is a typical case where the instability is everywhere convective (Huerre \& Monkewitz 1990). The flow is globally stable, but strongly amplifies incoming perturbations, and so behaves like a noise-amplifier (Chomaz 2004). A similar behaviour is observed for the parameters used in figure 3(c), the increase of confinement now being the cause of the flow stabilization.

Such spatio-temporal diagrams can be constructed for a wide range of parameters, in order to determine the stable and unstable regions on a $(-\Lambda, h)$ plane. The results are shown in figure 5. According to intuition, an increase of the velocity ratio, by means of the inner flow velocity, for example, tends to render the flow convectively unstable and thus globally stable. The influence of confinement is, however, more complex. The highest velocity ratios sustaining global instability are obtained for 


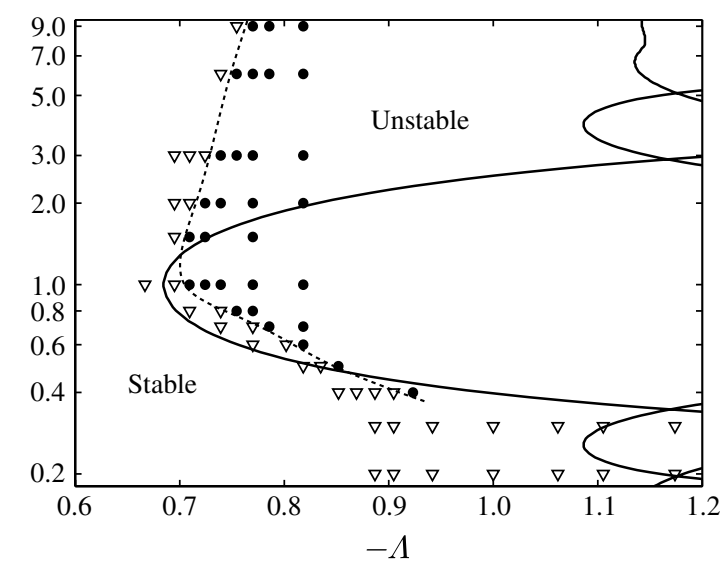

FIGURE 5. Stability diagram on the $(-\Lambda, h)$ plane for $R e=100$. The circles and triangles represent, respectively, globally unstable and globally stable configurations. The continuous line depicts the limit obtained by Juniper (2006) for an inviscid flow. In order to avoid confusion, not all simulated configurations have been represented.

$h \approx 1$, when the inner and the outer flows have the same thickness, while stronger and weaker confinements tend to stabilize the flow. This counterintuitive fact was previously predicted by Juniper (2006), as seen in figure 5, where his theoretical limit obtained for inviscid flows is reproduced (continuous line). Note that, according to the recent study of Rees \& Juniper (2010), the theoretical curve prevailing for the viscous instability of a top-hat profile at $R e=100$ is likely to be shifted to higher values of the velocity ratio.

According to Juniper's predictions, weaker co-flows are required to destabilize the flow at both strong and weak confinements, $h \ll 1$ and $h \gg 1$. Whereas Juniper predicted that counterflow was required for instability at $h>2.5$, our calculations show a weaker stabilization, with global instability even observed with consequent co-flow $(-\Lambda>0.76)$ at $h=9$. Note also that for very strong confinement, $h<0.4$, globally unstable flows were not encountered, in contrast to Juniper's inviscid predictions. Thus, we can conclude that viscosity plays a significant role for this Reynolds number. A higher Reynolds number is therefore analysed in the next subsection to get closer to the inviscid case studied by Juniper (2006).

\section{2. $R e=500$}

As for $R e=100$, the stability of the wake flows can be determined as a function of the velocity ratio and the confinement parameter by inspection of spatio-temporal diagrams. In addition to the typical behaviours depicted in figure 4 for $\operatorname{Re}=100$, where the flow is either stable or settles into a nonlinear limit cycle and displays a steady front (a situation that we will refer to as von Kármán instability, or VKI), we have also observed much more complicated behaviours for certain parameter settings. A typical example is provided by the spatio-temporal diagram depicted in figure 6 for $h=6$ and $\Lambda=-0.600$. Although the wave front is not advected towards the outlet, it is not fixed in space and in time, but vacillates around a mean position, displaying in this particular case a periodic low frequency modulation. This behaviour is in strong contrast with spatio-temporal diagrams typical of VKI instability (figure $4 a$ ) where the front is steadily fixed at an upstream location. We will refer to it as front vacillation instability (FVI). 


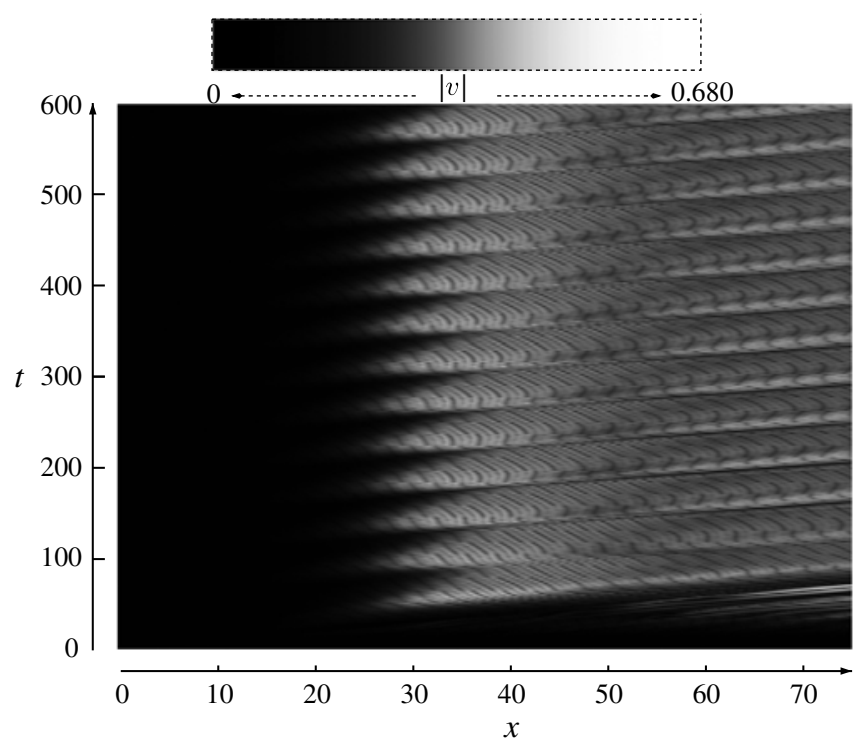

FIGURE 6. Spatio-temporal diagram of the absolute value of the velocity vertical component $v$ at $y=0$ for $h=6, \Lambda=-0.600$ and $R e=500$. The instability persists in the domain, but is characterized by a low frequency modulation.

\subsubsection{Vacillations}

Figure 7(a) shows time Fourier spectra of a vertical velocity probe $v(x=23.15, y=$ $0, t)$ for three different configurations. All of them are unstable, as seen in figure 9, but the weak confinement/weak co-flow case $(h=6, \Lambda=-0.6)$ is characterized by the FVI. The stronger confinement case, $h=1$, presents only a main high frequency, $f \approx 0.2$. It depicts a classical self-sustained nonlinear global mode instability (VKI), similar to that observed in figure $4(a)$, with the front wave fixed in space and time. For $h=6, \Lambda=-0.667$, the flow first displays a transient low frequency modulation before reaching a limit cycle. The spectral analysis, carried out on the established state, reveals peaks located at odd multiples of the main frequency.

For $h=6, \Lambda=-0.6$, one observes a broadened spiky peak, resulting from the low frequency modulation observed in figure 6 through an enriched beating mechanism between several equispaced frequency peaks. The gap between two consecutive peaks equals $\Delta f \approx 0.025$, which corresponds to a modulation period $T \approx 40$, as revealed from figure 6 . The observed modulations are reminiscent of a Benjamin-Feir-like sideband instability (Stuart \& DiPrima 1978) of the nonlinear wave prevailing in the downstream region of the flow.

The first issue is whether these vacillation modes are a robust feature or if they are highly sensitive to discretization, box length or outflow boundary conditions. We have checked the grid-convergence by using values of $N_{y}$ as high as $N_{y}=270$. The amplitude and period of the low frequency modulation are slightly modified, depending on the value of $N_{y}$, but the modulation is robust and persists in the domain. We have checked the influence of the exit condition on this feedback mechanism: the instability is still present, displaying the same low frequency modulation, as shown in figure $7(b)$. Furthermore, the location of the oscillating wave-front turns out to vary within a few per cent as the length or exit conditions are modified. While this excludes 

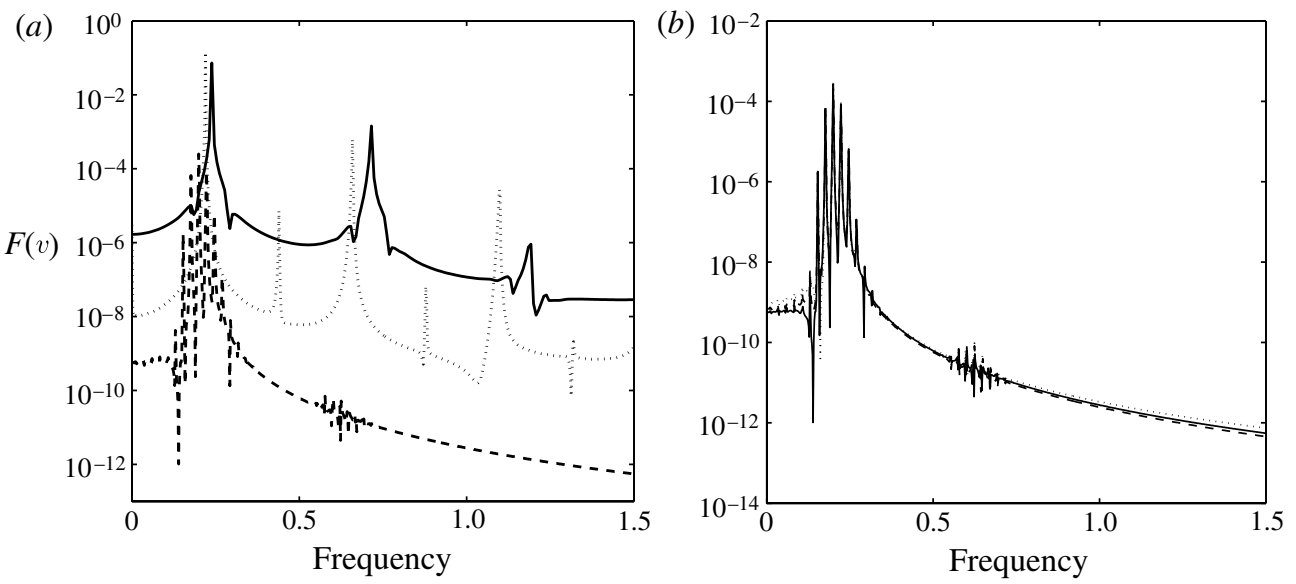

FiguRE 7. (a) Time Fourier spectra of $v(x=23.15, y=0)$ for three different unstable configurations at $R e=500$. Continuous line, $h=6, \Lambda=-0.667$; dotted line, $h=1$, $\Lambda=-0.667$; dashed line, $h=6, \Lambda=-0.600$. The latter configuration shows a low frequency modulation. The case with stronger confinement $h=1$ only shows the main high frequency, $f \approx 0.2$. (b) Time Fourier spectra for $h=6, \Lambda=-0.600$ and $R e=500$. Continuous line, $L=75$; dotted line, $L=101.5$; dashed line, $L=75$ plus an additional sponge subdomain. The low frequency modulation persists when varying the domain length or the outflow treatment. Note that for the $y$-axis the scale is modified between panels $(a)$ and $(b)$.

a numerical instability due to the outlet conditions, it does not clearly identify the governing feedback mechanisms at play.

\subsubsection{Front location}

While the spectral signatures of VKI and FVI significantly differ, as discussed above, they can also be discriminated based upon the location of the front. For small enough velocity ratio, the flows show a VKI-type instability with a front located in vicinity of the inlet. As the velocity ratio is increased, the steady front slowly migrates downstream until the sudden formation of a vacillating front located much further downstream. This scenario is readily observed in figure 8 , which displays, for $h=2$, the front locations in the $(x, t)$ plane for several values of the velocity ratio. The location of the front $x_{f}(t)$ is defined as the first grid-point where the $y$-component of the velocity on the centreline reaches the value of $v\left(x_{f}, 0, t\right)=10^{-3}$. As the VKI/FVI transition is passed, the front location experiences an important jump downstream as it simultaneously starts to vacillate. We have used in this study the velocity ratio at which this jump takes place as the threshold for VKI/FVI transition and monitored its dependence as a function of the confinement ratio.

\subsubsection{Stability diagram}

Figure 9 gathers the results for $R e=500$. As previously discussed, in the $(-\Lambda-h)$ plane three distinct regimes are discerned, characterized by the VKI (black solid circles), the FVI (grey solid squares) and by a stable flow (S) (empty triangles). The transition curve FVI/VKI depends on neither the size of the domain L nor the outlet boundary condition. This is, however, less clear for the FVI/S transition, which may be sensitive to the computational domain length $L$, although, as discussed in $\$ 3.2 .1$, the FVI is a robust feature. 


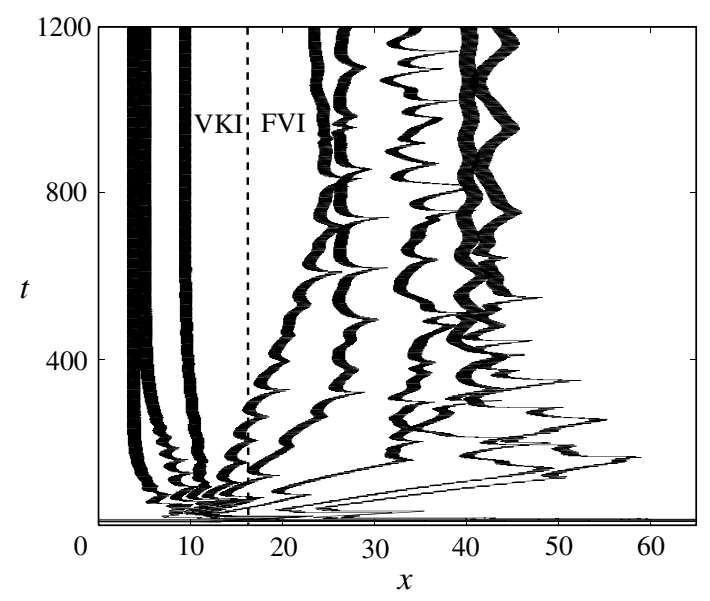

FIGURE 8. Wave front evolution for $h=2$ and different velocity ratio: from left to right, $\Lambda=\{-0.695,-0.68,-0.667,-0.653,-0.639,-0.600,-0.538,-0.504\}$. The jump between the third and fourth curves marks the limit between the VKI and FVI instabilities.

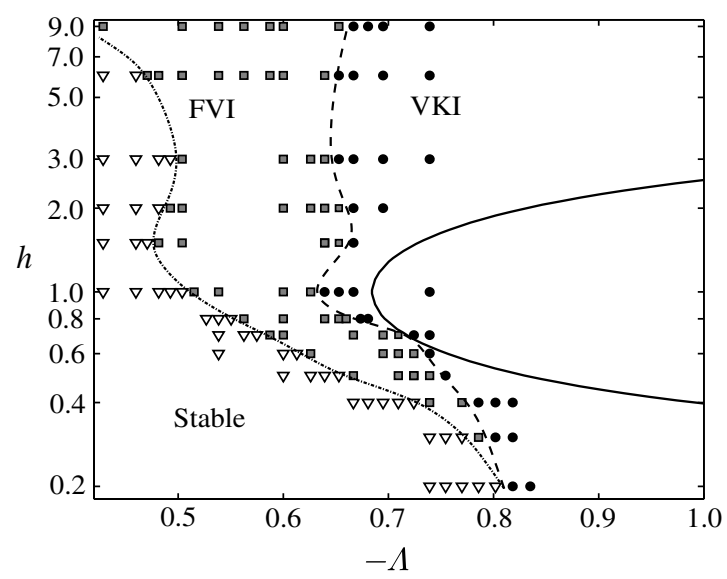

FIGURE 9. Stability diagram on the $(-\Lambda, h)$ plane, for $R e=500$. The circles, squares and triangles represent globally VKI unstable, FVI unstable and stable configurations, respectively. The continuous line depicts the limit obtained by Juniper (2006) for an inviscid flow. In order to avoid confusion, not all simulated configurations have been represented.

Comparing figures 9 and 5, the stabilizing role of the viscosity can be identified: a higher co-flow parameter is necessary to stabilize the flow at $R e=500$ than at $R e=100$. The transition curve is therefore shifted to lower values of $-\Lambda$. Another effect of lowering the viscosity is to extend the unstable region towards very strong confinements (for $h \leqslant 0.4$ ).

Furthermore, one can note that the FVI/VKI transition is rather close to Juniper's (2006) inviscid limit and bears some resemblance to the A/C instability threshold obtained by Rees \& Juniper (2010) and Biancofiore \& Gallaire (2011) by means of a local stability analysis of profiles including shear layer thickness. Since one can conjecture that a local analysis is not able to predict the instability connected 
to vacillations (see $\S 4.3$ for further details), it is more relevant to compare Juniper's threshold to the FVI/VKI limit rather than to the S/FVI one.

Analogous to figure 5 with $R e=100$, a maximum in the FVI/VKI transition curve for a confinement value of order one is also observed for $R e=500$, as predicted by Juniper's inviscid analysis. However, the FVI/VKI transition does not strictly follow the inviscid prediction pertaining to the inlet flow. The viscous transition curve at $R e=500$ is likely to be only slightly shifted to higher values of the velocity ratio, since in the presence of a free-slip boundary condition, the conclusion drawn from Monkewitz (1988) that the absolute/convective properties of unconfined wake instabilities becomes almost independent of $R e$ for $R e>200$ are expected to remain valid, a trend recovered by Rees \& Juniper (2010). It is noteworthy that some convectively unstable inlet velocity profiles, following the theoretical studies of Juniper, are seen to yield globally unstable flows. To be more precise, the inlet flow along the global stability transition line is always convectively unstable, excluding the flow dynamics to be governed by the inlet-driven global modes described by Couairon \& Chomaz (1999). There is no contradiction because the parallel flow $\boldsymbol{u}_{0}(x, y)$ is not a solution of the Navier-Stokes equations and the steady solution is a non-parallel flow $\boldsymbol{u}_{b}(x, y)$. The non-parallel character of the flow seems to play a key role and weakly non-parallel steep nonlinear global modes (Pier et al. 2001) are still plausible candidates for the VKI modes. This point is analysed in more detail in $\S 4$.

\subsection{Three-dimensional simulations}

The results shown in $\S 3.1$ and 3.2 were obtained for a two-dimensional domain and are therefore only fully justified if the influence of the third dimension is negligible. The objective of this section is to confirm that this is indeed the case. To this end, three-dimensional computations have been carried out in a domain of thickness $E=8$ equal to four times the inner wake width. This was suggested by the fact that for the wake of a cylinder, a channel depth equal to four times the inner wake width was found sufficient to see three-dimensional structures developing (Williamson 1996). For the initial condition, we have used the result obtained at the end of a two-dimensional computation, plus an additional perturbation. Two kinds of perturbation have been tried out, fully random or $E$-periodic, with an amplitude of $10^{-2}$. Note that the inlet boundary condition remains the two-dimensional velocity profile.

Figure 10(a) shows the spatio-temporal behaviour of the absolute value of the $z$-component of velocity, $|w|$, for $h=1, \Lambda=-0.667$ and $R e=500$ at a specific point of the $(y, z)$ plane. The periodic sinuous perturbation of the initial condition develops a three-dimensional effect on the flow, depicted by an increase of $|w|$. However, the three-dimensional perturbation is advected away and the flow comes back to a two-dimensional saturated state, or at least to a quasi-two-dimensional state such that the influence of the $z$ direction may be neglected. Using a random forcing rather than a periodic perturbation yields the same conclusion. It could be useful to remember that the configuration analysed in figure $10(a)$ is globally unstable. However, the same behaviour of $w$ has been observed for different three-dimensional simulations, varying confinement and velocity ratio, resulting in both stable and unstable configurations close to the limit shown in figure 9.

To confirm that the influence of the third dimension is negligible, we have considered a situation a priori favourable to the emergence of a three-dimensional flow. This is the case for $h=1, \Lambda=-1$, where $U_{1}=0$ guarantees no inner flow at the inlet of the channel. Figure 10(b) shows the corresponding spatio-temporal diagram, clearly showing a well-established three-dimensional flow. However, in the chosen 


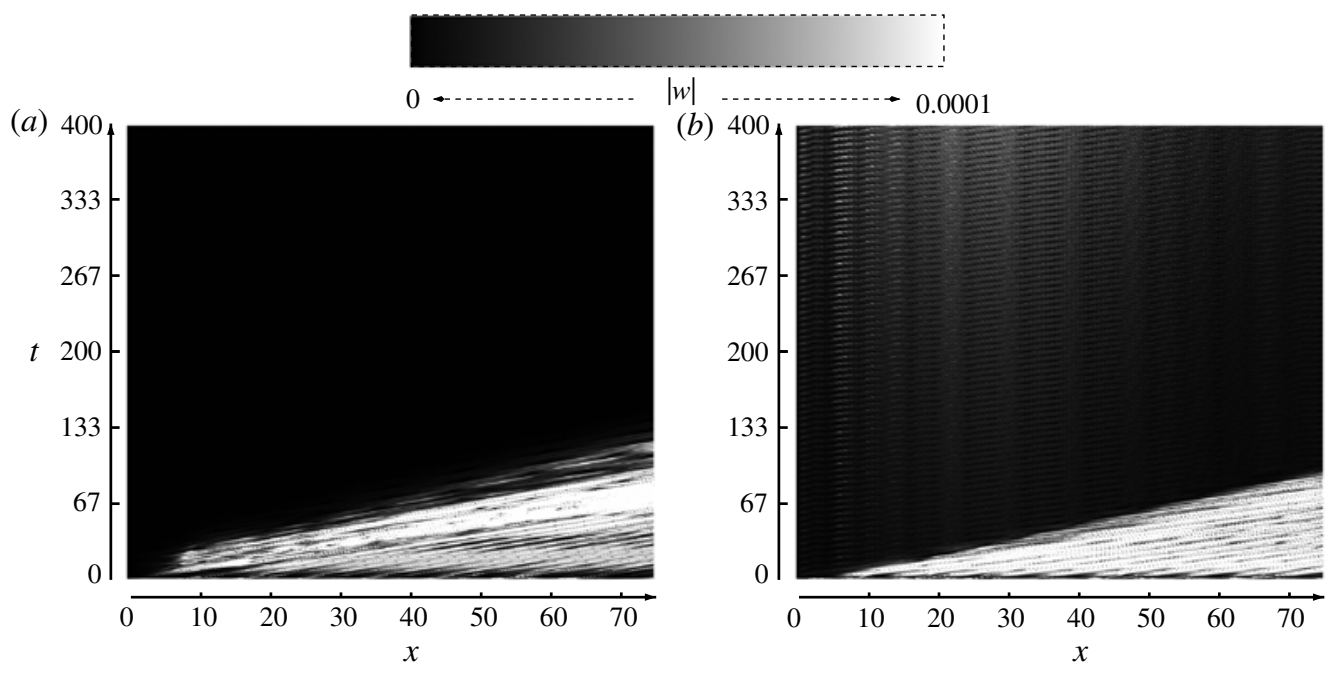

FIGURE 10. Spatio-temporal diagram of the absolute value of $z$-component of velocity $w$ for $R e=500, h=1$, and (a) $\Lambda=-0.667,(b) \Lambda=-1$, at $y=-1.335, z=-1$. In (a) the three-dimensional perturbation is advected away, while in $(b)$ the initial three-dimensional perturbation persists.

domain, with $L=75$, the amplitude of the spanwise component $w$ remains three orders of magnitude smaller than the mean streamwise velocity. Since the three-dimensional effects are detectable only far from the limit shown in figure 9, it is reasonable to assume that the two-dimensional approach is fully justified in the present study.

\section{Weakly non-parallel evolution of base flow}

\subsection{Influence of velocity ratio}

The base flow depicted in figure 2 illustrates that the velocity profile is modified by the effect of viscosity. Viscous effects tend to flatten the profile as a consequence of momentum diffusion and the flow is no longer parallel. This effect causes the velocity ratio $\Lambda(x)=\left(U_{\min }-U_{\max }\right) /\left(U_{\min }+U_{\max }\right)$ to vary along the domain. Since a constant velocity profile corresponds to $\Lambda=0$ and, remembering that for a wake we have $\Lambda<0$, it could be expected that $\Lambda$ would monotonically increase with $x$. Figure 2 , obtained for an unstable configuration, shows on the contrary that the velocity ratio $\Lambda$ may have a non-monotonous evolution along the domain: the velocity profile displays a clear velocity defect at $x=2$ indicating that, although the initial configuration at $x=0$ is a co-flow, a counterflow is present in a consistent part of the domain.

This wake retardation can be interpreted as the consequence of the advection-diffusion of the vorticity layer formed by the mixing layer. At the inlet, the vorticity distribution is infinitely thin but it then evolves downstream under the joint action of diffusion and advection, which leads to the development of boundary layers on both sides of the mixing layer. Since the advection is much stronger in the outer stream than in the inner wake, the external boundary layer is much thinner than the inner one and we may at leading order assume that the shear layer is only growing in the diffusion-dominated inner region while it does not penetrate in the advectiondominated outer region: see figure 11. Of course, this oversimplified assumption can 


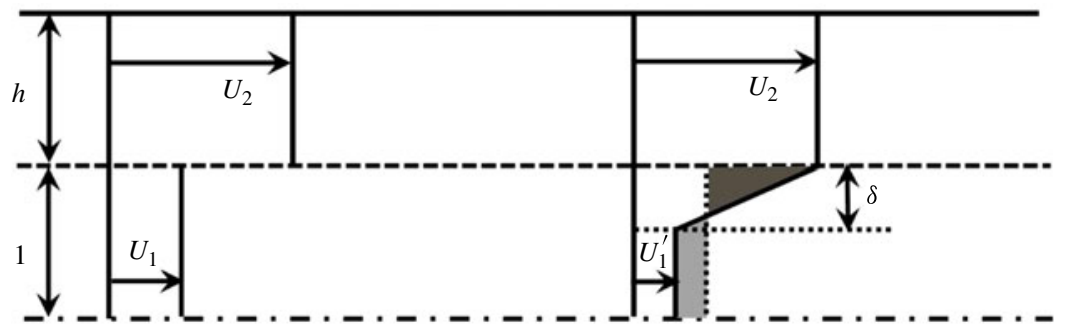

FIGURE 11. Physical mechanism at the origin of the wake deceleration.

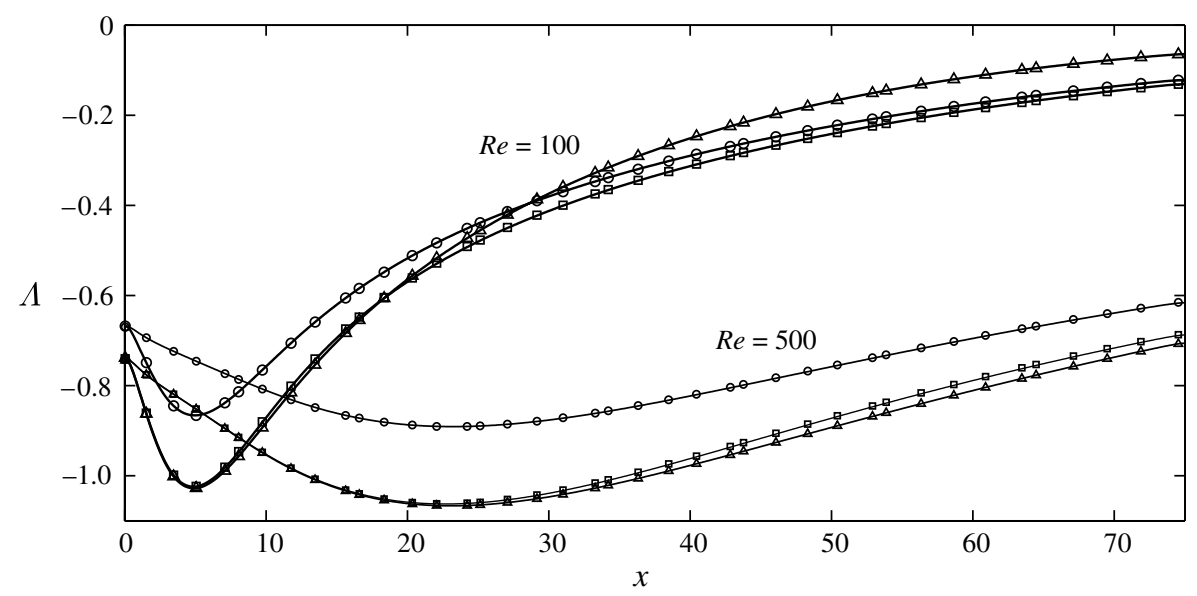

FIGURE 12. Evolution of the velocity ratio parameter $\Lambda$ along the domain. The bold lines represent the configurations for $R e=100$, the thin lines for $R e=500$ : $\square, h=1$ and $\Lambda=-0.739 ; \Delta, h=0.7$ and $\Lambda=-0.739 ; \circ, h=1$ and $\Lambda=-0.667$. The three configurations show a minimum $\Lambda$ at $x_{\min } \approx 5$ at $R e=100$ and at $x_{\min } \approx 25$ at $\operatorname{Re}=500$.

only remain valid while the shear layer thickness remains less than the inner stream width 1 . However, figure 11 readily makes clear that the central flow has to decelerate to ensure mass conservation.

Using the notation of figure 11 and applying the conservation of mass at a given streamwise position where the shear layer thickness equals $\delta$, one obtains

$$
U_{1}^{\prime}=U_{1} \frac{2-\delta U_{2} / U_{1}}{2-\delta} .
$$

This condition is satisfied if the two grey-shaded areas have equal surface. The velocity ratio also evolves accordingly,

$$
\Lambda^{\prime}=\frac{\Lambda}{1-\delta(1-\Lambda) / 2},
$$

and therefore decreases with increasing $\delta$.

Figure 12 shows the quantitative evolution of the velocity ratio $\Lambda(x)=\left(U_{\min }-\right.$ $\left.U_{\max }\right) /\left(U_{\min }+U_{\max }\right)$ along the streamwise direction for the three configurations considered in figure 3 and for the two different Reynolds numbers. One observes a minimum of the velocity ratio for all configurations. For $R e=100$, the minima are reached at $x_{\min } \approx 5$, while for $R e=500$ they are attained at $x_{\min } \approx 25$. These minima 


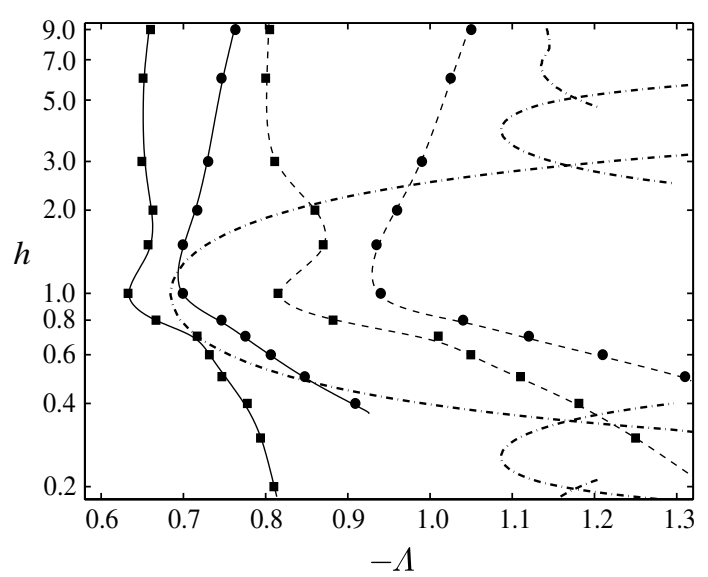

FIGURE 13. Comparison between the limit curves for the two different Reynolds numbers. The continuous lines depict the limit lines referred to the entry velocity ratio $\Lambda$, while dashed ones represent the limit referred to $\Lambda_{\min }$. Circles and squares depict the two Reynolds numbers $R e=100$ and $R e=500$, respectively. Juniper's (2006) limit curve for a parallel flow at $x=0$ is depicted with the dot-dashed line.

depend above all on the velocity ratio, while the confinement has no significant influence. When $x>x_{\text {min }}$, the velocity ratio starts to grow and tends to zero as $x$ goes to infinity. For a given flow, the minimal velocity ratio is expected to correspond to the locally most absolutely unstable region in the domain.

Thus, two different significant velocity ratios may be considered for a given configuration: the entry velocity ratio, $\Lambda(0)$, and the minimal velocity ratio, $\Lambda_{\min }$. Since the steep nonlinear front criteria of Pier et al. (2001) ensures a global instability as soon as an absolute instability is attained somewhere in the domain, we can analyse the limit curves obtained in figures 5 and 9 with respect to $\Lambda_{\text {min }}$. Figure 13 compares the limit curves for different Reynolds numbers as a function of both significant velocity ratios and the curve obtained by Juniper (2006) for an inviscid flow (continuous line). Assuming $R e=500$ is sufficiently large for viscous effects not to be dominant (see the discussion in $\S 3.2$ ), we would therefore expect Juniper's results to be closest to the limit curve for $R e=500$ and $\Lambda_{\text {min }}$, because this velocity ratio is expected to enhance at most the locally absolute instability for the non-parallel wake. This is not true, however, and the difference between the two curves is significant, invalidating at first sight a global stability prediction based on a steep nonlinear global mode anchored at the convective/absolute transition location.

\subsection{Influence of shear layer thickness}

Another interesting difference between our simulations and the analysis of Juniper (2006) may help to further explain this contrast: the absence of symmetry with respect to $h=1$ in the logarithmic scale for the limit curves obtained with DNS, while this symmetry is present in the top-hat wake model. At $R e=500$, the vacillations at weak confinements strongly break the symmetry (see figure 13), but the FVI/VKI limit, at $R e=500$, and the VKI/S one, at $R e=100$, are also not symmetric. The symmetry noticed by Juniper $(2006,2008)$ is due to the absence of a finite shear layer thickness, while the flows simulated here clearly exhibit a finite shear layer thickness, as shown in figure 2. Figure 14(a) provides the vorticity profiles of the base flow for $h=1$ and $\Lambda=-0.739$. At each $x$-location, the vorticity profile shows two peaks around $y= \pm 1$, 

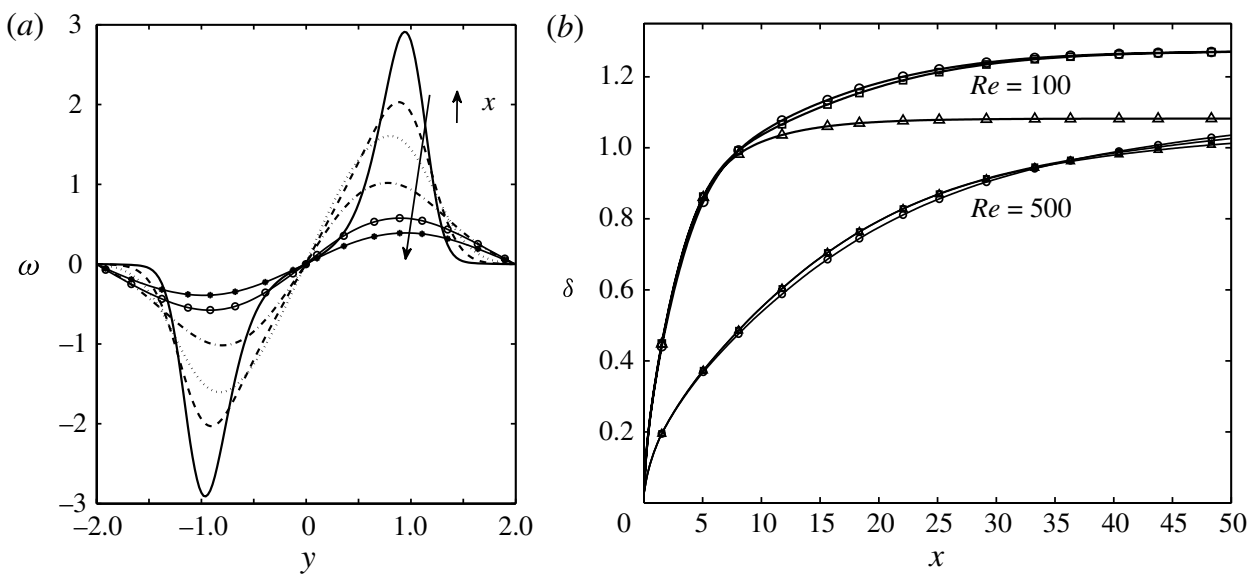

FIGURE 14. (a) Vorticity profiles of the base flow for $h=1, \Lambda=-0.739$ and $R e=100$. The profiles are given for $x=\{2.26,4.79,8.09,17.27,33.90,48.50\}$. As outlined by the arrows, the amplitudes are decreasing with respect to $x$. The vorticity profile at $x=0$, which is a regularization of a pair of opposite Dirac-delta functions, is not shown, its extrema being out of scale. $(b)$ Evolution of the shear layer thickness $\delta$ along the domain. The bold lines represent the configurations for $\operatorname{Re}=100$, and the thin lines for $\operatorname{Re}=500$ : $\square, h=1$ and $\Lambda=-0.739 ; \triangle, h=0.7$ and $\Lambda=-0.739 ; \circ, h=1$ and $\Lambda=-0.667$ (as in figure $9 b$ ).

indicating the inflection points of the corresponding velocity profiles, which are at the origin of the destabilization of the wake (Drazin \& Reid 1981).

The influence of the finite shear layer thickness on the strength of the inflection point instability has been addressed in the literature. While it is well known that the maximum temporal growth rate is proportional to the amplitude of the vorticity extremum, it has also been shown that the shear layer thickness $\delta=\left(U_{\max }-U_{\min }\right) /|\mathrm{d} U / \mathrm{d} y|_{\max }$ (Monkewitz 1988), which is inversely proportional to the vorticity extrema, also strongly modifies the absolute/convective properties of a parallel wake flow (Monkewitz 1988; Rees \& Juniper 2010). In particular, using a family of profiles parametrized by the shear layer thickness, Monkewitz (1988) has shown that there exists an optimal value of the shear layer thickness $\delta_{\text {opt }}(h=\infty) \approx 0.7$ for which absolute instability is maximal for inviscid unconfined wakes, and noticed a weak influence of $R e$ when $R e>100$. In a recent theoretical analysis, Biancofiore \& Gallaire (2011) have shown that this optimal value is indeed decreasing as confinement is introduced in Monkewitz's inviscid model. According to Biancofiore \& Gallaire (2011), for $h=1$ and $h=0.7$, one has $\delta_{\text {opt }}(h=1) \approx 0.2$ and $\delta_{\text {opt }}(h=0.7) \approx 0.15$, respectively. They also noted that the absolutely unstable character of the flow was strongly altered when $\delta$ was less than this optimal shear layer thickness.

Figure 14(b) shows the variations of the shear layer thickness $\delta$ in the streamwise direction. One observes monotonically increasing behaviour for all configurations. For both confinement ratios under consideration, $h=0.7$ and $h=1$, when the flow reaches the $\Lambda_{\text {min }}$-region, the shear layer thickness is much larger $(\delta \approx 0.85$, as seen in figure $14 b$ ) than $\delta_{o p t}(h), 0.15$ and 0.2 , respectively. The local velocity profile does not reach simultaneously the velocity ratio and shear layer thickness best promoting absolute instability. Thus, rather than the direct influence of viscosity on the parallel absolute/convective transition threshold, this mismatch of optimal $\Lambda$ and $\delta$ may elucidate the difference between our $R e=500 \mathrm{FVI} / \mathrm{VKI}$ transition curve and the theoretical absolute/convective limit relative to the minimal velocity ratio $\Lambda_{\min }$. 

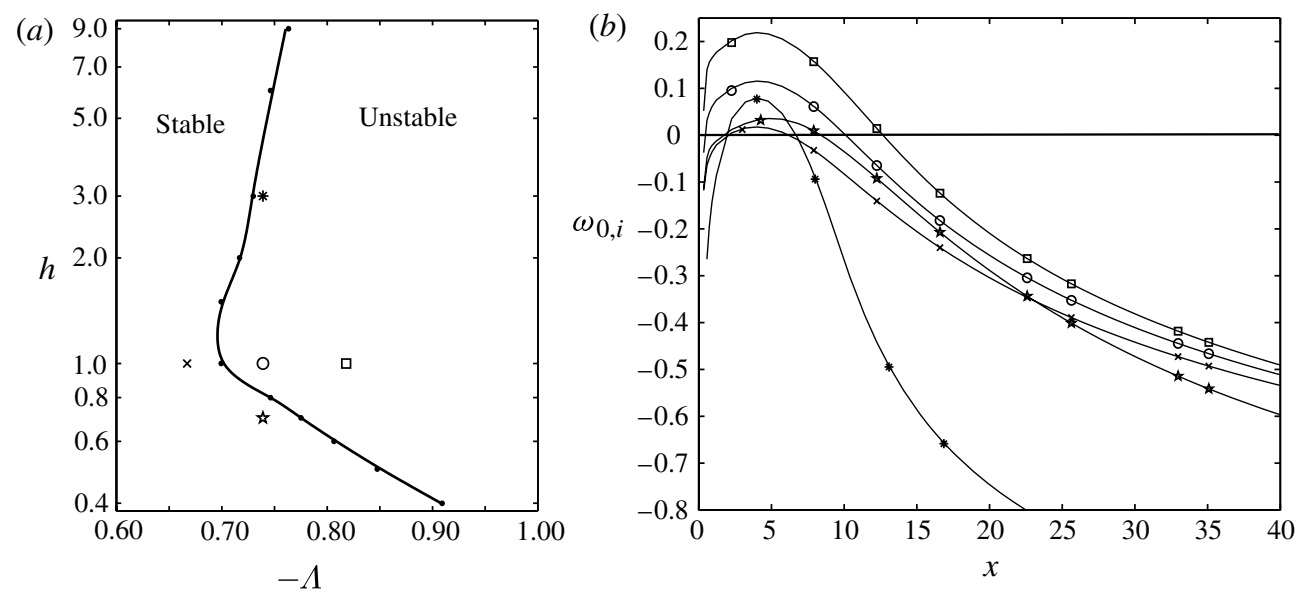

FIGURE 15. (a) Location of the considered configurations with respect to global unstable/stable limit curve for $R e=100: \circ, h=1$ and $\Lambda=-0.739 ; \square, h=1$ and $\Lambda=$ $-0.818 ; \times, h=1$ and $\Lambda=-0.667 ; \star, h=0.7$ and $\Lambda=-0.739 ; *, h=3$ and $\Lambda=-0.739$. (b) Streamwise variations of the absolute growth rate $\omega_{0, i}$ for such configurations. The numerical error bound on the absolute growth rate is $5 \%$.

\subsection{Local stability analysis}

In order to confirm this conjecture, we examine the local stability of several velocity profiles extracted from the base flow at different axial locations. Given a normal mode ansatz $\exp (\mathrm{i}(k x-\omega t)$, with $\omega$ for the complex frequency and $k$ for the complex wavenumber, the spatio-temporal stability analysis is based on the resolution of the Orr-Sommerfeld equation (Drazin \& Reid 1981), which can be discretized and rearranged into the form of a generalized eigenvalue problem (Rees \& Juniper 2010) and solved using MATLAB's function eig (MathWorks). The discretization is conducted on the same collocation points $y_{i}$ as those used in the DNS, i.e. the Chebyshev grid-points with the same mapping. In order to ensure the convergence of the procedure, different numbers of collocation points $N_{y}$ were tested, between 80 and 130. Dominant eigenvalues are affected by an error less than $5 \%$. A local dispersion relation, relating the complex frequency $\omega$ to the local complex wavenumber $k$, is thereby obtained.

As reviewed in Huerre \& Rossi (1998), the spatio-temporal stability analysis consists of finding the absolute mode corresponding to the admissible saddle point of highest imaginary part of the frequency $\omega_{i}$ in the complex wavenumber $k$-plane. The value of the imaginary part of frequency at the saddle point is called the absolute growth rate $\omega_{0, i}$, and it determines the nature of the local instability of the velocity profile: convective if $\omega_{0, i}<0$ or absolute if $\omega_{0, i}>0$. The iterative numerical procedure introduced by Deissler (1987) was used to determine the evolution of $\omega_{0, i}(x)$ with respect to the streamwise coordinate.

Figures 15(a) and 16(a) depict, for $R e=100$ and $R e=500$, the position in the plane $(-\Lambda, h)$ of the configurations for which local stability analyses were conducted, while the streamwise variations of the corresponding absolute growth rate $\omega_{0, i}(x)$ are shown in figures $15(b)$ and $16(b)$, respectively.

For $R e=100$, the globally unstable configurations, depicted by asterisks, circles and squares, have a significant absolutely unstable region (with positive $\omega_{0, i}$ ). An absolutely unstable region exists also for globally stable flows (marked by the cross 

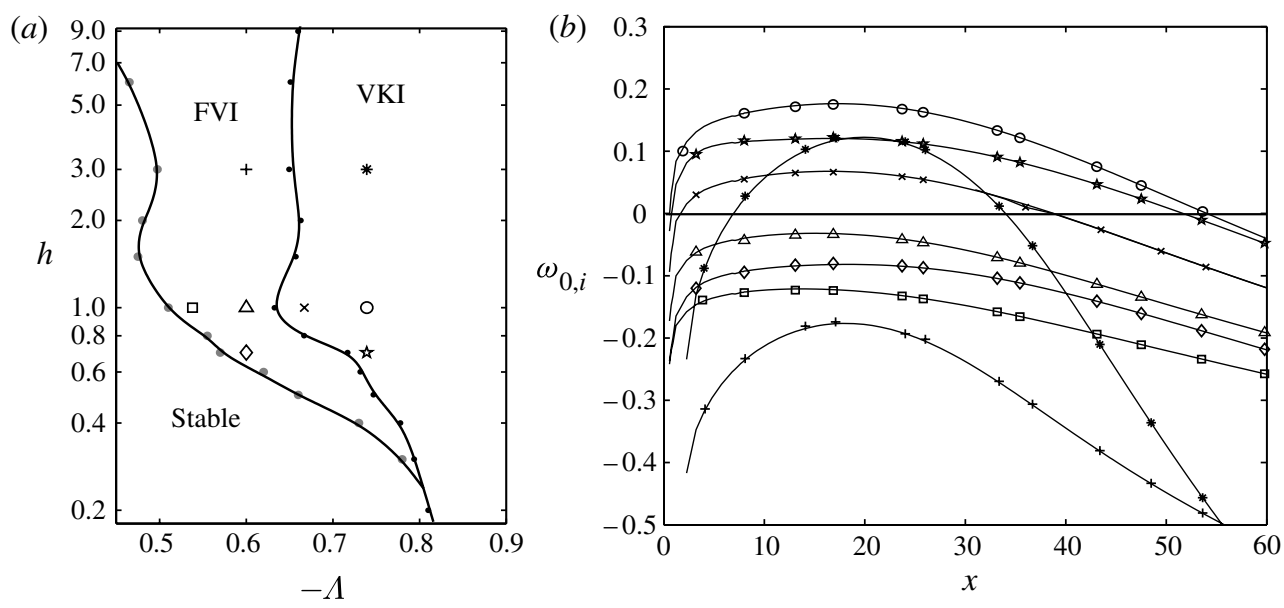

FIGURE 16. (a) Location of the considered configurations with respect to global unstable/stable limit curve for $R e=500: \circ, h=1$ and $\Lambda=-0.739 ; \times, h=1$ and $\Lambda=$ $-0.667 ; \triangle, h=1$ and $\Lambda=-0.600 ; \square, h=1$ and $\Lambda=-0.538 ; \star, h=0.7$ and $\Lambda=-0.739$; $\diamond, h=0.7$ and $\Lambda=-0.600 ; *, h=3$ and $\Lambda=-0.739 ;+, h=3$ and $\Lambda=-0.600$. (b) Streamwise variations of the absolute growth rate $\omega_{0, i}$ for such configurations. The numerical error bound on the absolute growth rate is $5 \%$, except for the profiles close to the inlet where it is still less than $10 \%$.

and star), with, however, both a less extended absolute region and a lower maximum value of absolute growth rate than those obtained for globally unstable configurations. This illustrates that the existence of a local region of absolute instability of finite extent is not a sufficient condition for global instability. The flow being far from parallel at $R e=100$, the local analysis cannot predict accurately its global stability under the weakly non-parallel flow assumption. The qualitative trends are, however, correctly captured.

Conversely, for $R e=500$, only the globally VKI flows present a region for which positive absolute growth rates are found. This shows that the local stability analysis seems to correctly predict the appearance of the VKI in this lower-viscosity case. This is not surprising, since flows at $R e=500$ can be considered weakly non-parallel and thus closer to the asymptotic limit of weakly non-parallel approximation, imposed by the local stability analysis.

However, note that the maximum value of $\omega_{0, i}$ is negative for all globally FVI configurations. The front vacillation instability is therefore observed when the flow is convectively unstable throughout the domain and cannot be predicted by the steep nonlinear global mode theory. This shows that the existence of a finite region of absolute instability is not a necessary condition for global instability of FVI type.

Furthermore, for the two flow cases we have analysed, the shedding frequencies obtained from DNS, resorting to the Fourier analysis already described in $\$ 3.2$ (see figure 7), are $\omega=1.28$ for the case $R e=100, h=1, \Lambda=-0.739$ and $\omega=1.38$ for $R e=500, h=1, \Lambda=-0.667$. Both frequencies have been evaluated at the same position of the domain, $x=23.15$. In view of the theoretical limit curve (Juniper 2006), the inlet profiles are convectively unstable. Hence, these frequencies have to be compared with the absolute frequency $\omega_{0, r}$ at the onset of absolutely instability region, as predicted by Pier et al. (2001): $\omega_{0, r}=1.49$ at $x=0.5$ for $R e=100$ (see figure 15b) and $\omega_{0, r}=1.44$ at $x=2.25$ for $R e=500$ (see figure $16 b$ ). The frequency predictions 
are thus accurate to within $14 \%$ and $4 \%$, respectively, showing the quantitative predictive power of linear local spatio-temporal stability theory to determine nonlinear global stability properties of weakly non-parallel flows at moderate Reynolds numbers.

\section{Conclusions and perspectives}

In this paper the global stability of non-parallel wakes, solutions of the Navier-Stokes equations, is studied by varying the velocity ratio $\Lambda$, the confinement ratio $h$ and the Reynolds number $R e$. We have constructed a stability diagram for $R e=100$ and $R e=500$ in order to analyse the effect of viscosity on such flows. These diagrams show the destabilizing influence of a moderate confinement (order one) which are qualitatively analogous to the theoretical studies of Juniper (2006).

The main difference from Juniper's results is the strong instability at weak confinements and sustained co-flow for $R e=500$, which takes the form of a vacillating front. An increase in viscosity, as analysed at $R e=100$, stabilizes the flow and quenches this instability, thus yielding moderate confinement to be the most unstable flow configuration, as predicted by Juniper. The discrepancies between Juniper's theory and results of DNS have been analysed in light of the non-parallel character of the base flow. A shear layer thickness is created by the viscous diffusion which modifies the base flow velocity profile, thus allowing the velocity ratio to vary along the domain. Using local velocity profiles extracted from the base flow at several streamwise locations, we have conducted a linear local stability analysis and determined the absolutely and convectively unstable regions. The local stability analysis turns out to give satisfactory predictions, specially for $R e=500$, for which the base flow is closer to conditions of parallelism. However, the existence of a region of absolute instability was shown to be neither a sufficient nor a necessary condition for global instability. The vacillating instability, in particular, could not be related to local properties.

In order to better understand the physical mechanism involved in the phenomena described in this paper, changing the sidewall conditions in the DNS may be important. Varying the boundary conditions at the sidewalls may indeed help us to understand the mechanisms of vacillations, which are enhanced by weak confinement and low viscosity. In order to bridge the gap to real flow applications of wakes exiting from injectors, the influence of confinement at large Reynolds number is also worth studying. In particular, by means of large-eddy simulations (LES), one could see how the transition to turbulence, or the strength of turbulence itself, i.e the mixing, are modified by confinement.

The calculations have been carried out on the computer of Mésocentre SIGAMM of the OCA (Observatoire de la Côte Azur) and on the NEC-SX8 supercomputer of IDRIS (project 094055). J.-M. Lacroix is warmly acknowledged for his kindness and technical support. J.-M. Chomaz, P. Meliga and J. Hoeppfner are warmly acknowledged for stimulating discussions.

\section{REFERENCES}

Azaiez, M., Fikri, A. \& Labrosse, G. 1994 A unique grid spectral solver of the $n$ D Cartesian unsteady Stokes system: illustrative numerical results. Finite Elem. Anal. Des. 16 (3-4), 247-260.

BiAnCOFiore, L. \& Gallaire, F. 2011 Influence of shear layer thickness on the stability of confined two-dimensional wakes. Phys. Fluids 23, 034103.

Borchers, W., Forestier, M.-Y., Kräutle, S., Pasquetti, R., Peyret, R., Rautmann, R., Ross, N. \& SABBAH, C. 1998 A parallel hybrid highly accurate elliptic solver for viscous 
flow problems. In Numerical Flow Simulation I (ed. C. Breitsamter \& E. H. Hirschel). pp. 3-24. Vieweg.

Chomaz, J.-M. 2004 Transition to turbulence in open flows: what linear and fully nonlinear local and global theories tell us. Eur. J. Mech. B 23, 385-399.

Chomaz, J.-M. 2005 Global instabilities in spatially developing flows: non-normality and nonlinearity. Аnnu. Rev. Fluid Mech. 37, 357-392.

Chomaz, J.-M., Huerre, P. \& RedeKopp, L. G. 1991 A frequency selection criterion in spatially developing flows. Stud. Appl. Math. 84, 119-144.

Couairon, A. \& Chomaz, J.-M. 1999 Fully nonlinear global modes in slowly varying flows. Phys. Fluids 11, 3688-3703.

Cousin, L. \& PASQuetTi, R. 2004 High-order methods for the simulation of transitional to turbulent wakes. In Advances in Scientific Computing and Applications, pp. 133-143. Sciences Press.

Coutanceau, M. \& Bouard, R. 1977 Experimental determination of the main features of the viscous flow in the wake of a circular cylinder in uniform translation. Part 1 . Steady flow. $J$. Fluid Mech. 79, 231-256.

Davis, R. W., Moore, E. F. \& Purtell, L. P. 1984 A numerical-experimental study of confined flow around rectangular cylinders. Phys. Fluids 27, 3688-3703.

DeIssler, R. J. 1987 The convective nature of instability in plane Poiseuille flow. Phys. Fluids 30, 2303-2305.

Drazin, P. G. \& ReID, W. H. 1981 Hydrodynamic Stability. Cambridge University Press.

Forestier, M.-Y., PAsquetti, R. \& PeYRet, R. Computations of three-dimensional wakes in stratified fluids. In Proceedings of Computational Fluid Dynamics Conference ECCOMAS 2000, Barcelona, Spain. 2000.

Gallaire, F., Ruith, M., Meiberg, E., Chomaz, J.-M \& Huerre, P. 2006 Spiral vortex breakdown as global mode. J. Fluid Mech. 549, 71-80.

GILL, G. S. 1978 A qualitative technique for concentric tube element optimization, utilizing the factor (dynamic head ratio-1). AIAA paper 16-18.

HAmmond, D. A. \& RedeKOPP, L. G. 1997 Global dynamics of symmetric and asymmetric wakes. J. Fluid Mech. 331, 231-260.

Hannemann, K. \& OeRtel, H. 1989 Numerical simulation of the absolutely and convectively unstable wake. J. Fluid Mech. 199, 55-88.

HeAley, J. J. 2007 Enhancing the absolute stability of a boundary layer by adding a far-away plate. J. Fluid Mech. 579, 151-168.

Healey, J. J. 2009 Destabilizing effects of confinement on homogeneous mixing layers. J. Fluid Mech. 623, 241-271.

Huerre, P. \& Monkewitz, P. A. 1990 Local and global instabilities in spatially developing flows. Annu. Rev. Fluid Mech. 22, 473-537.

Huerre, P. \& Rossi, M. 1998 Hydrodynamics instabilities in open flows. In Hydrodynamics and Nonlinear Instabilities (ed. C. Godrèche \& P. Manneville), pp. 81-294. Cambridge University Press.

JUNIPER, M. P. 2006 The effect of confinement on the stability of two-dimensional shear flows. J. Fluid Mech. 565, 171-195.

JUNIPER, M. P. 2007 The full impulse response of two-dimensional jet/wake flows and implications for confinement. J. Fluid Mech. 590, 163-185.

JUNIPER, M. P. 2008 The effect of confinement on the stability of non-swirling axisymmetric jets and wakes. J. Fluid Mech. 605, 227-252.

JUniper, M. P. \& CANDEL, S. 2003 The stability of ducted compound flows and consequences for the geometry of coaxial injectors. J. Fluid Mech. 482, 257-269.

KосH, W. 1985 Local instability characteristics and frequency determination of self-excited wake flows. J. Sound Vib. 99, 53-83.

LAsheras, J. C. \& Hopfinger, E. J. 2000 Liquid jet instability and atomization in a coaxial gas stream. Annu. Rev. Fluid Mech. 32, 275-308.

Leib, S. J. \& Goldstein, M. E. 1986 The generation of capillary instabilities on a liquid jet. $J$. Fluid Mech. 168, 479-500.

Lesshafft, L. \& Huerre, P. 2007 Linear impulse response in hot round jets. Phys. Fluids 19, 024102 . 
Loiseleux, T., Chomaz, J.-M. \& Huerre, P. 1998 The effect of swirl on jets and wakes: linear instability of the Rankine vortex with axial flow. Phys. Fluids 10, 1120-1134.

MAdAy, Y., PATERA, A. T. \& RonQuis, E. M. 1990 An operator-integration-factor splitting method for time-dependent problems: application to incompressible fluid flow. J. Sci. Comput. 5 (4), 263-292.

Marquillie, M. \& Ehrenstein, U. 2003 On the onset of nonlinear oscillations in a separating boundary-layer flow. J. Fluid Mech. 490, 169-188.

Meliga, P., Sipp, D. \& Chomaz, J.-M. 2010 Elephant modes and low-frequency unsteadiness in a high Reynolds number, transonic afterbody wake. Phys. Fluids 21, 054105.

Minguez, M., Pasquetti, R. \& Serre, E. 2008 High-order large-eddy simulation of flow over a simplified car model. Phys. Fluids 20, 095101.

Monkewitz, P. A. \& NGuyen, L. N. 1987 Absolute instability in the near-wake of two-dimensional bluff bodies. J. Fluids Struct. 1, 165-184.

Monkewitz, P. A. 1988 The absolute and convective nature of instability in two-dimensional wakes at low Reynolds numbers. Phys. Fluids 31, 999-1006.

Pasquetti, R., Bwemba, R. \& Cousin, L. 2008 A pseudo-penalization method for high Reynolds number unsteady flows. Appl. Numer. Math. 58, 946-954.

PAsquetTI, R. 2010 Temporal/spatial simulation of the stratified far wake of a sphere. Comput. Fluids 40, 179-187.

PEYRET, R. 2001 Spectral Methods for Incompressible Viscous Flow, Applied Mathematical Sciences, vol. 148. Springer.

Pier, B. \& Huerre, P. 2001 Nonlinear self-sustained structures and fronts in spatially developing wake flows. J. Fluid Mech. 435, 145-174.

Pier, B., Huerre, P. \& Chomaz, J.-M. 2001 Bifurcation to fully nonlinear synchronized structures in slowly varying media. Physica D 148, 49-96.

REeS, S. J. \& JUniper, M. P. 2010 The effect of confinement on the stability of viscous planar jets and wakes. J. Fluid Mech. 656, 309-336.

Rehimi, F., Aloui, F., Ben Nasrallah, S., Doubliez, L. \& Legrand, J. 2008 Experimental investigation of a confined flow downstream of a circular cylinder between two parallel walls. J. Fluids Struct. 24, 855-882.

Sabbah, C. \& PAsquetti, R. 1998 A divergence-free multi-domain spectral solver of the Navier-Stokes equations in geometries of high aspect ratio. J. Comput. Phys. 139, 359-379.

SAHin, M. \& Owens, R. G. 2004 A numerical investigation of wall effects up to high blockage ratios on two-dimensional flow past a confined circular cylinder. Phys. Fluids 16, 1305-1320.

Sevilla, A., Gordillo, J.-M. \& Martinez-BazAn, C. 2002 The effect of the diameter ratio on the absolute and convective instability of free coflowing jets. Phys. Fluids 14, 3028-3038.

Shair, F., Grove, A., Petersen, E. \& Acrivos, A. 1963 The effect of confining walls on the stability of the steady wake behind a circular cylinder. J. Fluid Mech. 17, 546-550.

StUART, J. T. \& DiPrima, R. C. 1978 The Eckhaus and Benjamin-Feir resonance mechanisms. Proc. R. Soc. Lond. A 362, 27-41.

Tammisola, O., Lundell, F., Schlatter, P., Wehrfritz, A. \& Södereberg, L. D. 2011 Global linear and nonlinear stability of viscous confined plane wakes with co-flow. J. Fluid Mech. 675, 397-434.

Triantafyllou, G. S., Triantafyllou, M. S. \& Chryssostomodis, C. 1986 On the formation of vortex streets behind stationary cylinders. J. Fluid Mech. 170, 461-477.

Triantafyllou, G. S. \& Karniadakis, G. 1990 Computational reducibility of unsteady viscous flow. Phys. Fluids A 2, 653-656.

Williamson, C. H. K. 1996 Vortex dynamics in the cylinder wake. Annu. Rev. Fluid Mech. 28, 477-539.

YU, M. H. \& Monkewitz, P. A. 1990 The effect of non-uniform density on the absolute instability of two-dimensional inertial jets and wakes. Phys. Fluids A 2, 1175-1181.

XU, C. J. \& PASQuetti, R. 2001 On the efficiency of semi-implicit and semi-Lagrangian spectral methods for the calculation of incompressible flows. Intl J. Numer. Meth. Fluids 35, 319-340. 